\title{
Partial Colectomy
}

National Cancer Institute

\section{Source}

National Cancer Institute. Partial Colectomy. NCI Thesaurus. Code C51887.

Surgical removal of part of the colon. 